\title{
Potenciales provocados auditivos en niños con riesgo neonatal de hipoacusia ${ }^{1}$
}

\author{
Saúl Garza Morales, ${ }^{2}$ Adrián Poblano, ${ }^{2}$ Alicia Robledo Galván ${ }^{3}$ \\ y Luis Alberto Fernández Carrocera ${ }^{4}$
}

\begin{abstract}
RESUMEN Los potenciales provocados auditivos del tallo cerebral (PPATC) son un método sencillo y no invasor de evaluación de la función auditiva, que se utiliza ampliamente en niños para detectar tempranamente hipoacusia. Entre abril de 1992 y mayo de 1994, se estudiaron 400 niños mexicanos que presentaban, al menos, un factor de riesgo neonatal de hipoacusia. La media de la edad de los niños estudiados fue 6,6 meses y la media de la edad gestacional al nacer, 35,1 semanas. El 51\% de ellos fueron tratados con amikacina. Se registraron 1427 factores de riesgo (3,5 por niño), entre los que predominaron la exposición a ototóxicos, la hiperbilirrubinemia y el peso al nacer menor de $1500 \mathrm{~g}$. En 27\% se encontraron alteraciones auditivas de tipo periférico y en $13 \%$, ausencia de respuesta a estímulos auditivos. El bajo peso y la menor edad gestacional al nacer, la concentración máxima de bilirrubina en el suero, la presencia de sepsis, la hemorragia subependimaria o intraventricular, la ventilación mecánica y la exposición a ototóxicos se asociaron significativamente con la presencia de hipoacusia grave o profunda.
\end{abstract}

Los potenciales provocados auditivos del tallo cerebral (PPATC) representan un método sencillo y no invasor para evaluar la función auditiva. Esta prueba se utiliza muy a menudo con objeto de detectar tempranamente la hipoacusia y alteraciones en la conducción neural de la vía auditiva en niños (1). La técnica no requiere la cooperación del paciente y es fácilmente repro-

\footnotetext{
1 Próximamente se publicará en esta revista una versión en inglés de este artículo.

2 Instituto Nacional de Perinatología, Departamento de Neurología Perinatal, Secretaría de Salud, Montes Urales 800, Loma de Virreyes, Delegación Miguel Hidalgo, CP 11000, México DF, México. La correspondencia debe enviarse al Dr. Garza Morales a la dirección anterior.

3 Centro Médico "20 de noviembre", Departamento de Enseñanza, Instituto de Seguridad y Servicios Sociales para los Trabajadores del Estado, México DF, México.

4 Instituto Nacional de Perinatología, Departamento de Seguimiento Pediátrico, Secretaría de Salud, México DF, México.
}

ducible $(2,3)$. Las primeras respuestas auditivas en neonatos aparecen entre las semanas 26 y 27 del embarazo $(4,5)$ y siguen temporalmente un "patrón de maduración" según el cual las latencias e intervalos interonda disminuyen y las amplitudes de los PPATC aumen$\tan (6)$.

La utilidad de esta prueba en recién nacidos para detectar alteraciones auditivas se ha comprobado en casos de premadurez (7), meningitis bacteriana (8), hiperbilirrubinemia (9), asfixia neonatal (10) y exposición prenatal al plomo (11). Además, se considera una prueba indispensable cuando existen factores de riesgo de alteraciones auditivas neonatales (12) (cuadro 1).

El objetivo del presente estudio fue describir el tipo y la frecuencia de alteraciones de los potenciales provocados auditivos del tallo cerebral (PPATC) en una población de 400 niños mexicanos que tenían al menos un factor de riesgo neonatal de hipoacusia.

\section{MATERIALES Y MÉTODOS}

Entre abril de 1992 y mayo de 1994 se analizaron los resultados de los potenciales provocados de 400 niños (en total se exploraron 800 oídos) que cumplieron los siguientes criterios de inclusión: haber nacido en el Instituto Nacional de Perinatología (México DF), radicar en el Valle de México (área metropolitana de la ciudad de México), tener, cuando menos, un factor de riesgo neonatal de hipoacusia (12), y haber aceptado participar en el Proyecto de Detección de Alteraciones Neuropsicológicas de Origen Perinatal (PDANDOP). En el estudio también se incluyeron niños que nacieron antes de abril de 1992, que participaban en el 
CUADRO 1. Factores de riesgo de hipoacusia en neonatos

Historia familiar de hipoacusia congénita Infección congénita perinatal: toxoplasma, rubéola, citomegalovirus, herpes y otras Malformaciones de cabeza y cuello

Peso al nacer $<1500 \mathrm{~g}$

Hiperbilirrubinemia con signos indicativos de exanguinotransfusión

Exposición a fármacos ototóxicos

Meningitis bacteriana

Apgar a los 5 minutos $<3$

Ventilación asistida durante más de 10 días

Sospecha de síndromes asociados con

hipoacusia: Waardenburg, Usher, etc.

Hemorragia subependimaria o intraventricular

Septicemia

Fuente: ref. 12.

PDANDOP, que no habían sido estudiados anteriormente con la prueba de potenciales provocados y que cumplían los criterios de inclusión consignados más arriba.

Todos los niños con resultados anormales en dicha prueba ingresaron en un programa de vigilancia. En dicho programa se repitieron los estudios y exploraciones después de 3 meses, se evaluó el desarrollo del lenguaje y se les realizaron pruebas de confirmación de la hipoacusia mediante audiometría tonal y logoaudiometría después de los 3 años de edad. En el presente trabajo solo se analizan los resultados de los estudios de potenciales provocados que se realizaron la primera vez.

Los potenciales provocados se estudiaron durante el sueño fisiológico mediante estimulación monoaural, con clicks de polaridad alterna y durante un período de adaptación previo al registro de 2 minutos de estimulación y aumentando progresivamente la intensidad $30 \mathrm{~dB}$ hasta llegar al nivel de audición de $80 \mathrm{~dB}$ (nivel de audición, presión sonora de 20 micropascales) en que se inició la promediación. El estímulo fue de 100 microsegundos y la tasa de repetición, de 11 estímulos/segundo. Los clicks se liberaron a través de audífonos TDH-49 en un cuarto aislado especialmente diseñado para este propósito. El oído contralateral se enmascaró con ruido blanco 30
dB por debajo de la intensidad del estímulo. La actividad eléctrica cerebral se registró con electrodos de disco de oro colocados en las derivaciones $\mathrm{Cz}(+)$, A1 y A2, después de limpiar la zona con alcohol-acetona y de aplicar gel conductor. El oído estudiado fue negativo y el contralateral, tierra (13).

La señal procedente de los electrodos se mantuvo por debajo de 4 kiloOhms y se utilizó para alimentar a una computadora. Los filtros pasabanda se colocaron entre 300 y $3000 \mathrm{~Hz}$ a fin de reducir el registro de otras señales biológicas (electrocardiograma y electromiograma). El tiempo de análisis posterior a la estimulación fue de 10 milisegundos. En total se promediaron 2024 estímulos y este proceso se repitió por lo menos una vez para asegurar la reproducibilidad de la respuesta. Luego de obtener el registro, se realizaron promediaciones, reduciendo la intensidad del estímulo por pasos de $20 \mathrm{~dB}$ hasta no obtener respuesta $\mathrm{y}$, posteriormente, por pasos ascendentes de $10 \mathrm{~dB}$ hasta conseguir una visualización clara y reproducible solo de la onda V (umbral electrofisiológico). Con un cursor se midió la latencia de las ondas I, III y V. La latencia de los intervalos interonda se obtuvo automáticamente con un programa computadorizado.

El resultado del estudio se presenta según los criterios de Stockard et al. (14) (cuadro 2). En el caso de niños prematuros, se tuvo en cuenta la edad posconcepcional o gestacional corregida (edad posnatal en semanas más edad gestacional al nacer).
Para realizar el análisis estadístico, se calcularon medias aritméticas, desviaciones estándar y recorridos de valores. Las medias se compararon mediante la prueba $t$ de Student (después de comprobar que las distribuciones de las variables eran normales y las varianzas, homogéneas), y las proporciones, mediante la prueba de ji cuadrado. El nivel de significación estadística seleccionado fue 0,05.

\section{RESULTADOS}

La media aritmética de la edad de los niños estudiados fue 6,6 meses (recorrido de valores: 1 día a 6 años). En el grupo de niños estudiados predominaron los menores de 6 meses (84\%). La media de la edad gestacional en el nacimiento fue 35,1 semanas (desviación estándar (DE) $\pm 3,5$ semanas; recorrido: 24 a 42). El 67\% de esos niños habían nacido antes de las 37 semanas. La media del peso al nacer fue $2034 \mathrm{~g}$ (DE = $870 \mathrm{~g}$; recorrido: 700 a $4575 \mathrm{~g})$ y con predominio de los nacidos con menos de 2000 g (234 casos). La concentración máxima de bilirrubina sérica indirecta durante el ingreso de los 265 niños evaluados fue $13,6 \mathrm{mg} / \mathrm{dL}(\mathrm{DE}=5,1$; recorrido: 1 a $30 \mathrm{mg} / \mathrm{dL})$. A 231 (57\%) niños se les administró amikacina endovenosa, a 97 (24\%), furosemida y a 2, gentamicina bajo la sospecha de que padecían septicemia neonatal.

En total se identificaron 1427 factores de riesgo (3,5 factores por niño). En $114(28 \%)$ niños solo se documentó un

\section{CUADRO 2. Clasificación de los potenciales provocados auditivos del tronco cerebral}

1. Normal. Cuando las latencias absolutas de las ondas I, III y V, los intervalos interonda I-III, III-V y $\mathrm{I}-\mathrm{V}$ se encuentran dentro del intervalo comprendido entre la media \pm 2 desviaciones estándar para la edad, y el umbral auditivo fue igual a $35 \mathrm{~dB}$ o menor.

2. Alteración periférica de tipo I. Cuando la latencia de la onda I y el umbral auditivo son altos.

3. Alteración periférica de tipo II. Cuando la onda I es de bajo voltaje o no existe y el umbral auditivo está elevado.

4. Ausencia de todos los componentes. Cuando no se logra identificar componente alguno.

5. Ausencia de componentes tardíos. Cuando no se pueden identificar las ondas III o V.

6. Incremento de los intervalos interonda.

Fuente: ref. 14 
CUADRO 3. Frecuencia de factores de riesgo de hipoacusia identificados en el estudio. ${ }^{\text {a }}$ Instituto Nacional de Perinatología, México, 1992-1994

\begin{tabular}{lc}
\hline \multicolumn{1}{c}{ Factor de riesgo } & $n$ \\
\hline Exposición a aminoglucósidos o furosemida & 235 \\
Septicemia neonatal & 231 \\
Ictericia que requiere fototerapia & 201 \\
Edad gestacional <34 semanas & 177 \\
Peso al nacer $<1500 \mathrm{~g}$ & 155 \\
Apgar al minuto < 3 & 134 \\
Ventilación asistida & 123 \\
Hemorragia subependimaria o intraventricular & 68 \\
Ictericia que requiere exanguinotransfusión & 46 \\
Infección congénita perinatal: & 15 \\
(rubéola 12, citomegalovirus 2, toxoplasmosis 1) & \\
Meningitis bacteriana & 13 \\
Exposición prenatal a antiepilépticos ${ }^{b}$ & 9 \\
Exposición prenatal a gentamicina & 8 \\
Hidrocefalia congénita & 5 \\
Hidranencefalia & 3 \\
Colpocefalia & 1 \\
Síndrome de Arnold-Chiari tipo 2 & 1 \\
Exposición prenatal a plomoc & 4 \\
Síndrome facio-aurículo-vertebral & 2 \\
Malformación auricular & 2 \\
Historia familiar de hipoacusia congénita & 1 \\
Craneosinostosis & 1 \\
Retraso del crecimiento intrauterino & 1 \\
Antecedente familiar de muerte súbita & 1 \\
Total & 127 \\
\hline potal & \\
\hline
\end{tabular}

a Un paciente pudo tener más de un factor de riesgo.

b Proyecto de estudio de los efectos de la exposición prenatal a antiepilépticos sobre los potenciales provocados auditivos del tallo cerebral (23).

c Proyecto de estudio prospectivo del plomo de la ciudad de México (10). y en el resto (59\%), 3 o más (cuadro 3).

Se detectaron anormalidades auditivas en $45 \%$ de los oídos estudiados, entre las cuales predominaron las periféricas $(27,5 \%)$ y la ausencia de todos los componentes $(13,4 \%)$ (cuadro 4, figuras 1 y 2). factor de riesgo, en 51 (13\%), 2 factores
Al analizar las anormalidades de los diferentes grupos de riesgo de hipoacusia, la mayor proporción de niños con anormalidades se observó en el grupo de los que padecían hemorragia subependimaria o intraventricular $(79 \%)$, meningitis bacteriana (76\%), ictericia y exanguinotransfusión (69\%)
CUADRO 4. Resultados obtenidos en la exploración de los 800 oídos. Instituto Nacional de Perinatología, México, 1992-1994

\begin{tabular}{lrr}
\hline \multicolumn{1}{c}{ Hallazgos } & No. & $\%$ \\
\hline Normal & 438 & 54,7 \\
Alteración periférica I & 120 & 15,0 \\
Alteración periférica II & 100 & 12,5 \\
Ausencia de componentes tardíos (III y V) & 3 & 0,4 \\
Reducción de la relación I/V & 9 & 1,1 \\
Intervalo I-V aumentado & 23 & 2,9 \\
Ausencia de todos los componentes & 107 & 13,4 \\
\hline
\end{tabular}

y en los que desarrollaron sepsis neonatal (68\%) (cuadro 5).

Por otro lado, se comprobó la existencia de una relación inversa entre la edad gestacional, el peso al nacer y la presencia de resultados anormales en los estudios de PPATC. En la figura 3 aparece, a modo de ejemplo, la relación entre la edad gestacional al nacer y la frecuencia de alteraciones auditivas.

A fin de analizar los factores de riesgo asociados con la ausencia bilateral de todos los componentes en el estudio de PPATC (grupo I, $n=48$ ), las características de los niños con este resultado se compararon con los restantes 352 participantes (grupo II). En el grupo I se detectó una cifra significativamente más elevada de factores de riesgo por niño que en el grupo II $\left(X^{2}=\right.$ 46,3; $P<0,001)$. Además, se detectaron diferencias significativas entre ambos grupos en cuanto al peso al nacer $(t=$ -3,94; $P<0,0001)$, edad gestacional $(t=$ $-4,66 ; \quad P<0,0001)$, concentración máxima de bilirrubina sérica $(t=-3,25$; $P<0,001)$, uso de amikacina $\left(X^{2}=\right.$ 41,90; $P<0,001)$, furosemida $\left(X^{2}=\right.$ $18,80 ; P<0,01)$, frecuencia de meningitis bacteriana $\left(X^{2}=31,22 ; P<0,001\right)$, hemorragia subependimaria $\mathrm{O}$ intraventricular $\left(X^{2}=27,66 ; P<0,001\right)$, y el empleo de ventilación mecánica $\left(X^{2}=\right.$ 26,09; $P<0,001$ ) (cuadro 6).

\section{DISCUSIÓN}

Los niños que padecen pérdida auditiva durante los primeros meses de vida y cuyos umbrales auditivos se encuentran por encima de $65 \mathrm{~dB}$ a menudo tienen un desarrollo lingüístico defectuoso. El éxito de la rehabilitación depende directamente de su detección y tratamiento tempranos (4). El elevado número de niños seleccionados en este estudio refleja la alta frecuencia de niños que tienen factores de riesgo de trastornos auditivos. Estos niños son dados de alta de unidades de terapia neonatal especializada y podrían beneficiarse si se sometieran a estudios de detección de hipoacusia antes de cumplir el primer año de vida (90\% de la población estudiada). 
FIGURA 1. Registro normal de potenciales provocados auditivos del tronco cerebral (PPATC) de un neonato cuya madre padeció rubéola durante el tercer trimestre del embarazo. (OD = oído derecho; $\mathrm{Ol}=$ oído izquierdo). Los trazos superiores muestran las principales ondas (I, III, V) a $80 \mathrm{~dB}$, y los inferiores, el umbral electrofisiógico a $30 \mathrm{~dB}$. Barras de calibración horizontal $=1$ miliseg; vertical $=0,16$ microvoltios. (Los estudios se muestran por duplicado para asegurar la reproducibilidad). Instituto Nacional de Perinatología, México, 1992-1994
OD

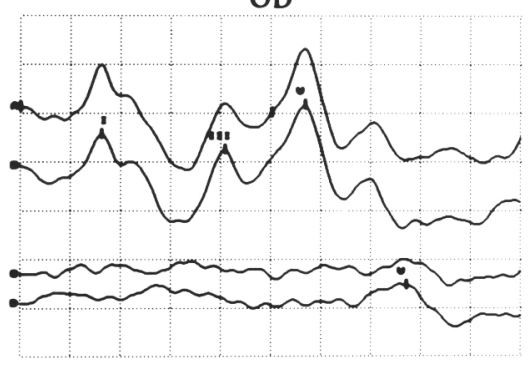

ol

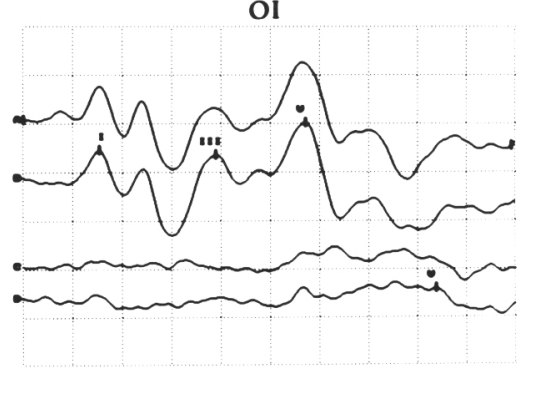

I
FIGURA 3. Relación entre el porcentaje de alteraciones auditivas y la edad gestacional al nacer. Instituto Nacional de Perinatología, México, 1992-1994

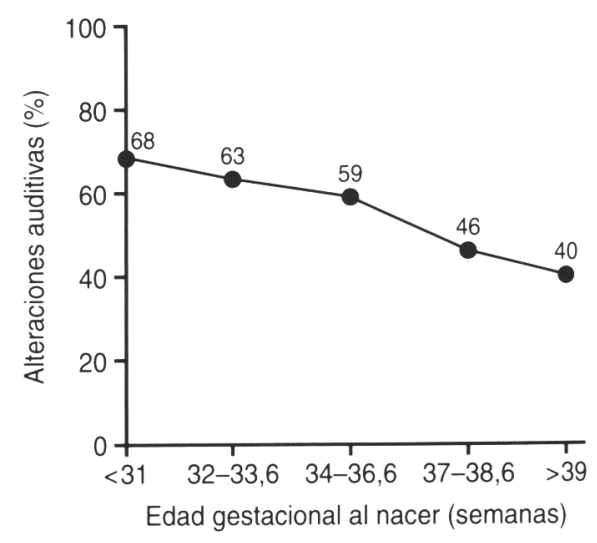

La mayoría de los pacientes estudiados nacieron antes de la semana 37 de gestación con un peso menor de $2000 \mathrm{~g}$ y requirieron la administración de fármacos potencialmente ototóxicos (amikacina, furosemida). Ello explica que en cerca de la mitad de los estudios realizados se identificaran anormalidades; como causas se han documentado la afectación coclear (en expuestos a furosemida y amikacina) $(15,16)$, disfunción tubárica (en los que recibieron ventilación mecánica) (17) y afectación de núcleos cocleares como consecuencia de hiperbilirrubinemia e hipoxia (17-19). Cabe resaltar la alta frecuencia de estudios en los que se han diagnosticado anormalidades en niños que desarrollaron hemorragia subependimaria o intraventricular, meningitis bacteriana, ictericia y septicemia, lo cual se explica por efecto sobre el nervio acústico (20), hemorragia coclear (21) y exposición a otros factores de riesgo (ventilación mecánica, aminoglucósidos, etc.).

La relación inversa entre la frecuencia de trastornos auditivos y la edad gestacional y el peso al nacer hacen suponer, en primer lugar, que las vías neurológicas auditivas inmaduras son especialmente susceptibles a estos trastornos. En segundo lugar, es posible que las secuelas auditivas se asocien más a menudo con la mayor 
CUADRO 6. Comparación de los factores de riesgo de los grupos de niños con ausencia bilateral de todos los componentes de los potenciales provocados auditivos del tronco cerebral (grupo I) y el resto de pacientes (grupo II). Instituto Nacional de Perinatología, México, 1992-1994

\begin{tabular}{lccc}
\hline \multicolumn{1}{c}{ Factores de riesgo } & $\begin{array}{c}\text { Grupo I } \\
(n=48)\end{array}$ & $\begin{array}{c}\text { Grupo II } \\
(n=352)\end{array}$ & $P$ \\
\hline Peso al nacer & $1637 \mathrm{~g}$ & $2087 \mathrm{~g}$ & $0,0001^{\mathrm{a}}$ \\
& \pm 721 & \pm 875 & \\
Edad gestacional & 33,1 & 35,3 & $0,001^{\mathrm{a}}$ \\
al nacer (semanas) & $\pm 2,9$ & $\pm 3,5$ & \\
Concentración sérica máxima de & 16,5 & 13,1 & $0,001^{\mathrm{a}}$ \\
bilirrubina indirecta & \pm 6 & $\pm 4,8$ & $\mathrm{NS}^{\mathrm{b}}$ \\
Apgar al minuto $<3$ & 17 & 117 & $\mathrm{NS}^{\mathrm{b}}$ \\
Infección congénita prenatal (TORCH) & 1 & 14 & $\mathrm{NS}^{\mathrm{b}}$ \\
Fototerapiac & 30 & 171 & $0,01^{\mathrm{b}}$ \\
Exanguinotransfusión & 12 & 34 & $0,05^{\mathrm{b}}$ \\
Sepsis neonatal & 34 & 191 & $0,001^{\mathrm{b}}$ \\
Meningitis bacteriana & 8 & 5 & $0,01^{\mathrm{b}}$ \\
Uso de amikacina & 47 & 170 & $0,01^{\mathrm{b}}$ \\
Uso de furosemida & 25 & 80 & $0,001^{\mathrm{b}}$ \\
HSE/IV & 21 & 47 & $0,001^{\mathrm{b}}$ \\
Ventilación mecánica & 27 & 86 & $0,001^{\mathrm{b}}$ \\
Más de 3 factores de riesgo & 42 & 126 & \\
\hline
\end{tabular}

a $t$ de Student.

b Prueba de $\chi^{2}$

c Todos los que fueron sometidos a exanguinotransfusión se expusieron a fototerapia.

Abreviaturas: NS: no significativo; TORCH : infección congénita perinatal por toxoplasma, rubéola, citomegalovirus o herpes; HSE/IV: hemorragia subependimaria o intraventricular.

supervivencia de los neonatos de bajo peso al nacer que están expuestos a múltiples factores potencialmente ototóxicos (22).
La ausencia de registro de potenciales a intensidades mayores de $80 \mathrm{~dB}$ del nivel de audición se asoció con alteración auditiva grave (14). Los fac- tores de riesgo asociados estadísticamente con este tipo de alteración permiten identificar un perfil de alto riesgo en neonatos. Estos factores son los siguientes: bajo peso al nacer, menor edad gestacional, ictericia intensa, haber recibido amikacina o furosemida, haber estado sometido a ventilación mecánica, hemorragia subependimaria o intraventricular y meningitis bacteriana (23).

La asociación estadística del mayor número de factores de riesgo con la presencia de hipoacusia profunda sugiere que los factores de riesgo mencionados pueden ejercer un efecto sinérgico, especialmente en niños con menor peso y menor edad gestacional al nacer. En estos niños las complicaciones son extremadamente frecuentes, porque permanecen en el hospital durante períodos muy prolongados. Los factores de riesgo enumerados se observan cada vez con más frecuencia en los niños dados de alta de las unidades de neonatología. Estos pacientes constituyen un grupo de alto riesgo de presentar trastornos auditivos. El problema social y económico planteado por los niños con hipoacusia, cuyo cuidado exige tecnología y personal especializado en rehabilitación (psicólogos, pedagogos, lingüistas), puede ser importante en el futuro.

\section{REFERENCIAS}

1. Picton TW, Taylor MJ, Durieux-Smith A. Brainstem auditory evoked potentials in pediatrics. En: Aminoff MJ, ed. Electrodiagnosis in clinical neurology. New York: Churchill Livingstone; 1992:537-569.

2. Jewett D, Williston JS. Auditory evoked farfields averaged from the scalp of humans. Brain 1971;94:681.

3. Dehkharghani F. Applications of electroencephalography and evoked potentials studies in the neonatal period. En: Sarnat HB, ed. Topics in neonatal neurology. Orlando: Grune Stratton; 1984:257-288.

4. Kennedy CR. The assessment of hearing and brainstem function. En: Eyre JA. The neuro- physiological examination of the newborn infant. London: Mac Keith Press; 1992:79-92.

5. Aicardi J. Disorders of hearing and vestibular function. En: Aicardi J, ed. Diseases of the nervous system in childhood. London: Mac Keith Press; 1992:1076-1092.

6. Stockad-Pope JE, Werner SS, Bickford RG Evoked potentials. En: Stockard-Pope JE, Werner SS, Bickford RG. Atlas of neonatal electroencephalography. New York: Raven Press; 1992: 53-92.

7. Smyth V, Scott J, Tudehope D. Auditory brainstem evoked response inter-peak latencies in very low birthweight infants. Int J Ped Otorhinolaryngol 1988;16:69-76.
8. Cohen BA, Schenk VA, Sweeney DB. Meningitis-related hearing loss evaluated with evoked potentials. Pediatr Neurol 1988;4:18-21.

9. Nakamura H, Takada S, Shimabuku R, Matsuo M, Matsuo T, Negishi H. Auditory nerve and brainstem responses in newborns infants with hyperbilirubinemia. Pediatrics 1985;75: 703-708.

10. Poblano A, Mendiola BH, Valdez $\mathrm{CH}$, et al. Potenciales provocados auditivos del tallo cerebral en recién nacidos de bajo y alto riesgo. Bol Med Hosp Infant Mex 1993;50: 551-556.

11. Rothenberg SJ, Poblano A, Garza-Morales S Prenatal and perinatal low level lead exposure 
alters brainsterm auditory evoked responses in infants. Neurotoxicology 1994;15:695-699.

12. Bergman I, Hirsch RP, Fria TJ, Shapiro SM, Holzman I, Painter MJ. Cause of hearing loss in the high-risk premature infant. $J$ Pediatr 1985;106:95-101.

13. Nuwer MR, Aminoff M, Goodin D, Matsuoka $S$, Mauguière F, Starr A, et al. IFCN recommended standards for brainstem auditory evoked potential: report of an IFCN committee. Electroenceph Clin Neurophysiol 1994;91: 12-17.

14. Stockard JE, Stockard JJ, Kleinberg F, Westmoreland BF. Prognostic value of brainstem auditory evoked potentials in neonates. Arch Neurol 1983;40:360-365.

15. Finitzio-Hieber T. Auditory brainstem response in assessment of infants treated with aminoglycoside antibiotics. En: Lerner SA, Matz GJ, Hawkins JE Jr, eds. Aminoglycoside ototoxicity. Boston: Little, Brown and Company; 1981:269-280.
16. Brown DR, Watcho JF, Sabo D. Neonatal sensorineural hearing loss associated with furosemide: a case-control study. Dev Med Child Neurol 1991;33:816-823.

17. Streletz LJ, Graziani LJ, Branca PA, Desai HJ, Travis SF, Mikaelian DO. Brainstem auditory evoked potentials in full-term and preterm newborns with hyperbilirubinemia and hypoxemia. Neuropediatrics 1986;17:66-71.

18. Salamy A, Eldrege L, Tooley HW. Neonatal status and hearing loss in high-risk infants. J Pediatr 1989;114:847-852.

19. Poblano A, Garza-Morales S, Ibarra-Puig J. Utilidad de los potenciales provocados auditivos del tallo cerebral en la evaluación del recién nacido. Bol Med Hosp Infant Mex 1995; 52:262-270

20. Jiang ZD, Liu XY, Wu YY, Zheng MS, Liu HC. Long-term impairments of brain and auditory functions of children recovered from purulent meningitis. Dev Med Child Neurol 1990;32: 473-480.
21. Spector GJ, Pettit WJ, Davis G, Strauss M, Rauchbach E. Fetal respiratory distress causing CNS and inner ear hemorrhage. Laryngoscope 1978;88:764-784.

22. McCormick MC. ¿Se ha incrementado la prevalencia de lactantes discapacitados al mejorar la supervivencia del lactante con peso muy bajo al nacer? Clin Perinatol 1993;1: 285-302. (Traducción al español realizada por la Editorial Interamericana.)

23. Jones KL, ed. Fetal trimethadione effects: Smith's recognizable patterns of human malformation. Philadelphia: WS Saunders Co; 1988:500-501.

Manuscrito recibido el 25 de octubre de 1995 y aceptado para publicación, tras revisión, el 6 de mayo de 1996.

ABSTRACT Auditory evoked potentials of the brain stem (AEPBS) provide a simple, noninvasive method of evaluating hearing function and have been widely used for early detection of hypoacusis in children. Between April 1992 and May 1994, a study was done of 400

\section{Auditory evoked potentials in children at neonatal risk for hypoacusis}

Mexican children who presented at least one neonatal risk factor for hearing impairment. The average age of the children studied was 6.6 months and their average gestational age at birth was 35.1 weeks. Just over half of them $(51 \%)$ had been treated with amikacin. The study found 1427 risk factors (3.5 per child), the most common ones being exposure to ototoxic substances, hyperbilirubinemia, and birthweight of less that $1500 \mathrm{~g}$. In $27 \%$ of the children, peripheral auditory changes were found, and $13 \%$ did not respond to auditory stimuli. Low birthweight and young gestational age at birth, high serum concentration of bilirubin, sepsis, subependymal or intraventricular hemorrhage, mechanical ventilation, and exposure to ototoxic substances were significantly associated with the presence of severe or profound hypoacusis. 